\title{
基于石墨烯及其复合物电极的电容去离子技术研究进展
}

\author{
冯爱虎，于云，宋力昕 \\ (中国科学院 上海硅酸盐研究所, 中国科学院特种无机涂层重点实验室, 上海 200050)
}

摘 要: 电容去离子技术是一种高效节能、绿色环保的脱盐方法, 通过施加静电场, 强制离子向两侧电极迁移, 使其 被电极表面产生的双电层吸附, 从而达到脱盐的目的。电容去离子技术的关键是高性能电极材料的制备, 要求具有 较高的比表面积、合理的孔径分布和良好的导电性。石墨烯具有较高的理论比表面积和优异的导电性，是一种理想 的电极材料。然而由于石墨烯的聚集效应，实际比表面积远远低于理论值，将石墨烯制备成三维网络结构或将石墨 烯与其他材料进行复合可以克服聚集效应，提高电极的脱盐性能。本文综述了基于石墨烯及其复合物电极的电容去 离子技术研究进展、存在的问题及应用前景。

关 键 词: 石墨烯; 电极; 电容去离子; 综述

中图分类号: TB383 文献标识码: A

\section{Research Progress of Graphene and Its Composites as Electrodes for Capacitive Deionization}

\author{
FENG Ai-Hu, YU Yun, SONG Li-Xin
}

(Key Laboratory of Inorganic Coating Materials, Shanghai Institute of Ceramics, Chinese Academy of Sciences, Shanghai 200050, China)

\begin{abstract}
Capacitive deionization is an energy-efficient and environment-friendly desalination method, which forces ionic species toward oppositely charged high-surface-area electrodes under an electric field to achieve the purpose of desalination. The key technology is to prepare electrode materials, which require high specific surface area, reasonable pore size distribution and excellent electrical conductivity. Graphene is a desired kind of electrode material used in capacitive deionization for its high specific surface area and wonderful conductivity. However, the actual specific surface area is far below the theoretical value due to the effect of aggregation of graphene. The three-dimensional graphene or the composite materials can overcome aggregation effect to improve the performance of electrode. The research progress of the capacitive deionization technology based on graphene and its composite electrode are reviewed in detail. The existing problems and application prospect are also objectively pointed out in this review.
\end{abstract}

Key words: graphene; electrodes; capacitive deionization; review

随着人类社会的快速发展，淡水资源危机成为 世界各国亟待解决的问题，水资源短缺已经成为制 约多国发展的主要因素。地球上 $98 \%$ 的水资源以海 水和咸水的形式存在, 不能供人类直接使用, 发展 绿色高效的海水脱盐技术将是解决淡水资源短缺问
题的重要途径 ${ }^{[1]}$ 。目前，广泛使用的海水淡化技术主 要包括: 反渗透法(Reverse osmosis, RO)、电渗析法 (Electro-dialysis，ED)和热蒸发法(Thermal evaporation, TE) 等 $^{[2]}$ 。但这些技术都有一定的局限性，普遍 存在能耗高，成本高及二次污染等问题。如反渗透 
技术和热蒸发技术需要高驱动能量, 能耗大, 脱盐 效率低, 且易产生二次污染 ${ }^{[3-4]}$; 而电渗析技术则需 较高的工作电压 ${ }^{[5]}$ 。因此, 迫切需要研发低成本、高 效率和环境友好的新型绿色脱盐技术。

电容去离子技术(Capacitive deionization, CDI) 具有工作电压低、成本低且无二次污染等优点, 是 一种较理想的海水淡化方法, 其历史可以追溯到 20 世纪 60 年代, Caudle 等 ${ }^{[6]}$ 使用多孔炭电极的流动电 容装置(Flow-through capacitor) 从碱性水中除去盐 分。随后, Johnaon 等 $^{[7]}$ 研究了不同的电极材料, 证 实离子在多孔碳电极上的选择性吸附, 并通过进一 步的理论研究, 提出了双电层吸附离子的概念。然 而直到 90 年代中期, 美国劳伦斯国家实验室的 Farmer 等 ${ }^{[8-9]}$ 制备出碳气凝胶电极后, 人们才更加 关注 CDI 技术。之后, 随着各种多孔电极材料的制 备, CDI 技术得到了快速发展。

一般来说, CDI 电极材料要求具有较高的比表 面积、合理的孔径分布和良好的导电性 ${ }^{[10]}$ 。高的比 表面积和合理的孔径分布可以提供更多的空位用于 吸附离子, 良好的导电性有利于提高离子的传输速 率, 提高电极的比电容。多孔碳材料均能满足 CDI 电极的上述条件, 是一种理想的 CDI 电极材料。目 前研究报道的碳电极材料主要包括: 活性炭 ${ }^{[11-12] 、 ~}$ 碳气凝胶 $[10,13]$ 、介孔碳 ${ }^{[14-15]}$ 、碳纳米管 ${ }^{[16-18]}$ 和石墨 烯 ${ }^{[19-21]}$ 。其中, 石墨烯是一种由碳原子以 $\mathrm{sp}^{2}$ 杂化轨道 组成的六角蜂巢型平面薄膜, 其比表面积 $\left(2600 \mathrm{~m}^{2} / \mathrm{g}\right)$ 和电导率(7200 S/m)远高于活性炭等其他碳材料 ${ }^{[22]}$ 。 因此, 在能量储存器件, 如超级电容器和锂离子电 池中有广泛的应用, 基于石墨稀电极的超级电容器 的容量可达到 $200 \mathrm{~F} / \mathrm{g}$ 以上 ${ }^{[23]}$ 。大量研究表明石墨 烯在 CDI 技术中存在着巨大应用潜力, 本文将详细 综述石墨稀及其复合物作为 CDI 电极的研究现状、 存在问题及应用前景。

\section{1 石墨烯 CDI 电极的研究}

\section{1 二维石墨烯 CDI 电极的研究}

石墨烯具有较大的比表面积和良好的导电性, 是一种理想的 CDI 电极材料。2009 年, 华东师范大 学 $\mathrm{Li}$ 等 ${ }^{[21]}$ 利用改进的 Hummer 法 ${ }^{[24-25]}$ 合成了氧化 石墨, 以水合肼为还原剂, 制备出石墨烯薄层, 并 首次将其应用于 $\mathrm{CDI}$ 电极。他们以 $\mathrm{NaCl}$ 为溶液, 对 该电极材料的离子吸附能力进行了研究, 发现电极 的电吸附量达到 $1.85 \mathrm{mg} / \mathrm{g}$, 初步证实石墨烯可以作为 CDI 电极材料。然而制备的石墨烯薄层的比表面积仅
为 $14.2 \mathrm{~m}^{2} / \mathrm{g}$, 远低于理论值，随后 $\mathrm{Li}$ 等 ${ }^{[17]}$ 通过改变 实验参数制备出比表面积为 $77 \mathrm{~m}^{2} / \mathrm{g}$ 的石墨烯, 说 明石墨烯的比表面积与制备方法有关。

南澳大学 Zou 等 ${ }^{[26]}$ 研究了 Hummer 法中硝酸和 硫酸的含量对石墨烯薄层比表面积的影响。研究发 现，当两者体积比为 1:1 时，制备的石墨烯比表面积 最大, 为 $222.1 \mathrm{~m}^{2} / \mathrm{g}$ 。但测试发现, 基于此种石墨烯的 CDI 电极的电吸附量仅为 $23.18 \mu \mathrm{mol} / \mathrm{g}(1.35 \mathrm{mg} / \mathrm{g})$, 低于 $1.85 \mathrm{mg} / \mathrm{g}$ 。说明电极的电吸附性能不完全由材 料的比表面积决定, 比表面积仅仅是决定电吸附性 能的一个因素。

利用氧化还原法制备石墨烯时，还原剂的选择 十分重要。上述方法中所用到的还原剂水合肼有毒, 而且可能会污染石墨烯。上海大学 Zhang 等 ${ }^{[27]}$ 利用 吡啶作为插入剂和分散剂，通过热解剥离的方法制 备出高分散性石墨烯薄层, 其厚度为 $2.2 \mathrm{~nm}$, 合成 工艺如图 1 所示。测试表明, 基于此种石墨烯的 CDI 电极具有良好的电化学性能。

Jia 和 Zou 等 ${ }^{[19,28-29]}$ 采用多步还原方法, 用多 种还原剂分步还原氧化石墨烯，成功制备出分散性 更好的石墨烯薄层, 其比表面积达到 $464 \mathrm{~m}^{2} / \mathrm{g}$ 。他 们首先使用铁粉进行初步还原, 然后进行磺化, 引 入 $-\mathrm{SO}_{3}^{-}$, 以阻止石墨烯薄层的聚合 ${ }^{[30]}$, 最后使用 水合肼进一步还原, 制备出分散性良好的石墨烯薄 层, 制备工艺如图 2 所示。研究表明, 基于此种石墨 烯的 CDI 电极的电吸附量达到 $8.6 \mathrm{mg} / \mathrm{g}$, 比电容达 到 $149.8 \mathrm{~F} / \mathrm{g}$, 电极性能远远好于之前报道。

综上所述, 将石墨烯用于 CDI 电极的研究已经 取得了一定的成绩，制备出了电吸附量较大的石墨 烯电极材料。不同还原法制备的石墨烯的电化学性 能不同，选择合适的实验条件可以得到性能优异的 石墨烯电极材料。但目前制备石墨烯的方法主要为 氧化还原法，石墨烯在氧化还原过程中往往存在

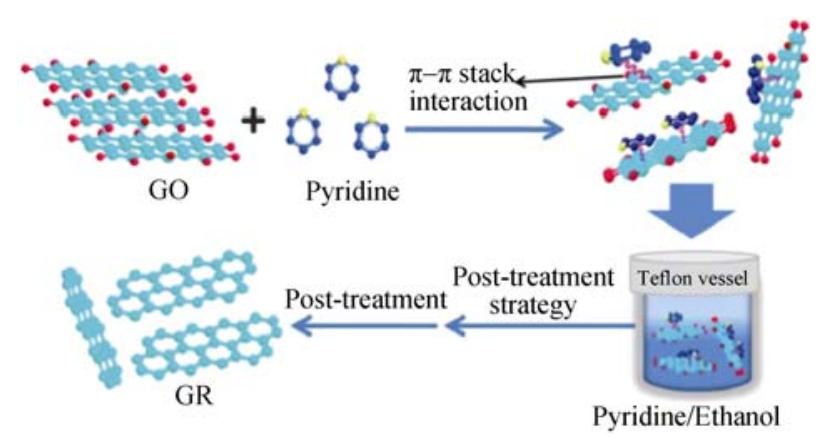

图 1 吡啶一热解法制备石墨烯的流程图 ${ }^{[27]}$

Fig. 1 Procedure of the GR preparation by pyridine-thermal strategy ${ }^{[27]}$ 


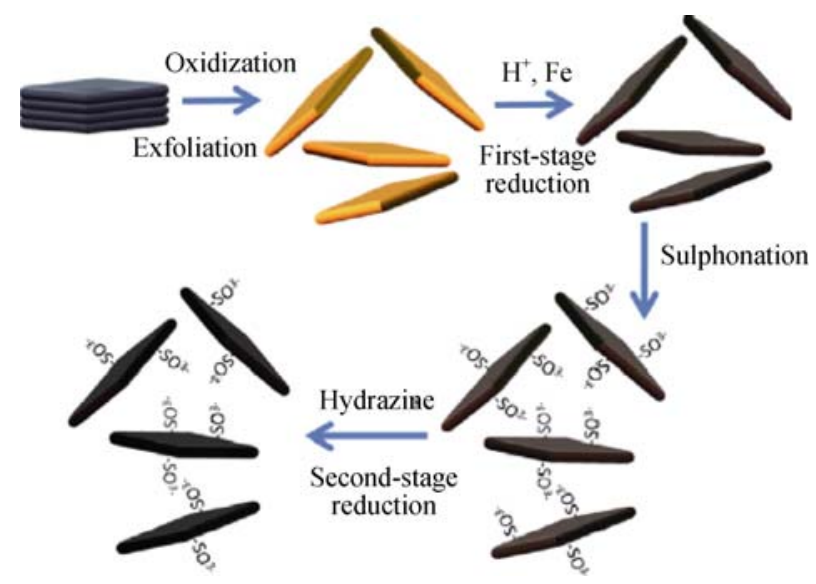

图 2 多步还原合成的石墨烯示意图 ${ }^{[19]}$

Fig. 2 Schematic of the multistep reduction synthesis of graphene $^{[19]}$

反应不完全和二维石墨烯聚集问题，严重影响离子 的传输速率和电极的导电性。此外, 聚集效应降低石 墨烯的比表面积, 进而影响电极的电化学性能 ${ }^{[1-32]}$ 。

\section{2 三维石墨烯 CDI 电极的研究}

与二维石墨烯纳米材料相比, 三维石墨烯材料
的组织结构更特殊，具有更大的比表面积和分布均 匀的孔径, 更适合作为 CDI 电极材料 ${ }^{[33-34]}$ 。

Wang 等 ${ }^{[35]}$ 利用 Worsley 报道的溶胶-凝胶法 ${ }^{[36]}$ 成功制备出三维石墨烯气凝胶(RGO-RF), 并将其应 用于 $\mathrm{CDI}$ 电极。 $\mathrm{N}_{2}$ 吸脱附等温线表明 RGO-RF 的比 表面积为 $406.4 \mathrm{~m}^{2} / \mathrm{g}$, 几乎是原二维石墨烯 $(R G O)$ 的 三倍, 而平均孔径由 $6.502 \mathrm{~nm}$ 减小到 $3.124 \mathrm{~nm}$, 更有 利于吸附 $\mathrm{NaCl}$ 离子。电极吸附性能测试表明, 基于 RGO-RF 的 CDI 电极的电吸附量和比电容分别为 $1.424 \mathrm{mg} / \mathrm{g}$ 和 $135.7 \mathrm{~F} / \mathrm{g}$ (扫描速度为 $10 \mathrm{mV} / \mathrm{s}$ ), 高于 $\mathrm{RGO}$ 的 $0.799 \mathrm{mg} / \mathrm{g}$ 和 $112 \mathrm{~F} / \mathrm{g}$, 表明石墨烯气凝胶具 有更好的电极性能。此外, 他们还进行了吸附动力学 研究, 发现离子的吸附主要为单层吸附, 满足朗缪尔 吸附等温曲线, 离子饱和吸附量为 $13.245 \mathrm{mg} / \mathrm{g}$ 。

Zhang 等 ${ }^{[37-38]}$ 分别以聚苯乙烯球(PS)和 $\mathrm{SiO}_{2}$ 球 作为骨架材料与氧化石墨水溶液均匀混合，经高温 裧烧, 氧化石墨热解剥离为三维状石墨烯, 部分高 分子聚合物炭化形成多孔碳，成功制备出三维多孔 石墨烯(3DMGA)和石墨烯基三维分级多孔碳材料 (3DGHPC), 合成过程如图 3 和图 4 所示。3DMGA

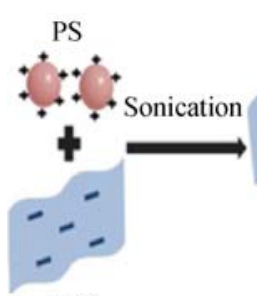

$\mathrm{GO}$

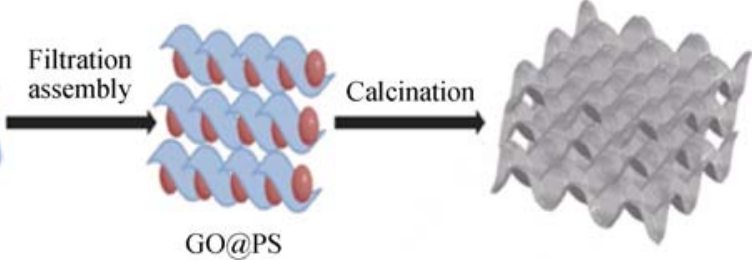

3DMGA

图 $33 \mathrm{DMGA}$ 的合成路线示意图 ${ }^{[37]}$

Fig. 3 Schematic illustration of the synthetic route for $3 \mathrm{D} \mathrm{MGA}^{[37]}$

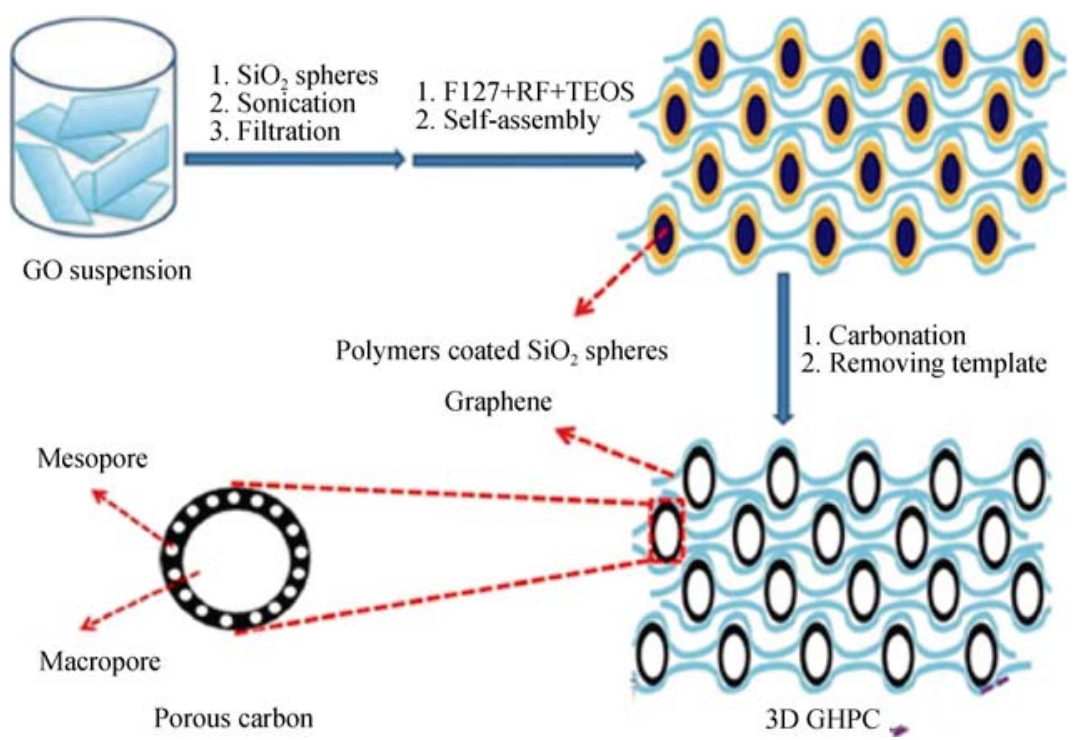

图 4 3DGHPC 的合成路线示意图 ${ }^{[38]}$

Fig. 4 Schematic illustration of the synthetic route for 3D GHPC ${ }^{[38]}$ 
和 3DGHPC 的比表面积分别为 $339 \mathrm{~m}^{2} / \mathrm{g}$ 和 $384.4 \mathrm{~m}^{2} / \mathrm{g}$, 高于之前报道的二维石墨烯薄层; 电吸附性能测试 表明基于这两种三维石墨烯的 CDI 电极的电吸附量 分别为 $3.9 \mathrm{mg} / \mathrm{g}$ 和 $6.18 \mathrm{mg} / \mathrm{g}$ 。说明三维石墨烯更适 合作 CDI 电极材料。之后, 他们又利用 PS 球和酚 醛树脂 $(\mathrm{PF})$ 等作为骨架材料, 制备出了一类新的三 维材料一石墨烯包覆的中空介质碳球 $(\mathrm{GHMCS})^{[39]}$, 合成路线如 5 图所示, 其比表面积为 $400.4 \mathrm{~m}^{2} / \mathrm{g}$, 约 为所用石墨烯薄层的 5 倍。

Zhang 等制备的三维石墨烯的电化学性能优异, 但制备工艺复杂，且成本较高，不利于大规模工业 生产。北京理工大学 Yang 等 ${ }^{\left[{ }^{[0]}\right.}$ 报道了一种更简便、 低成本的制备方法，如图 6 所示。他们将氧化石墨 烯溶液浸入到海绵中, 经 $900^{\circ} \mathrm{C}$ 煅烧, 海绵消失, 直接得到多孔三维石墨烯材料(STGS)。经测试, STGS 的比表面积为 $305 \mathrm{~m}^{2} / \mathrm{g}$, 基于 STGS 的 CDI 电
极的电吸附量和比电容分别为 $4.95 \mathrm{mg} / \mathrm{g}$ 和 $57 \mathrm{~F} / \mathrm{g}$ (扫 描速度为 $10 \mathrm{mV} / \mathrm{s}$ ), 相同情况下, 无海绵骨架石墨烯 电极的电吸附量和比电容仅为 $2.85 \mathrm{mg} / \mathrm{g}$ 和 $45 \mathrm{~F} / \mathrm{g}$ 。 此外, STGS 制备的电极显示更好的循环稳定性, 1000 次循环后，电极性能稳定在 $99 \%$ ，且离子脱 吸附时间较短, 仅为 $25 \mathrm{~min}$ 。最近, $\mathrm{Xu}$ 等 ${ }^{[41]}$ 将氧 化石墨烯溶液冷冻干燥，然后在氮气气氛下，经 $800^{\circ} \mathrm{C}$ 煅烧 $3 \mathrm{~h}$ 制备出另一种海绵状石墨烯(GS), 该 GS 材料的比表面积为 $356.0 \mathrm{~m}^{2} / \mathrm{g}$ ，气孔体积达 到 $1.51 \mathrm{~cm}^{3} / \mathrm{g}$ 。CDI 电极性能测试表明, 溶液初始 浓度为 $500 \mathrm{mg} / \mathrm{L}$ 时, 该 $\mathrm{GS}$ 的电吸附量为 $14.9 \mathrm{mg} / \mathrm{g}$, 为原石墨烯的 3.2 倍。此外, GS 电极的比电容测试 结果为 $205.2 \mathrm{~F} / \mathrm{g}$ (扫描速度为 $5 \mathrm{mV} / \mathrm{s}$ ), 远高于普通 石墨烯的 $117.31 \mathrm{~F} / \mathrm{g}$ 。

$\mathrm{NaOH} 、 \mathrm{KOH}$ 等强碱可用来激发碳材料，通过 刻蚀材料, 增加缺陷, 从而达到提高其比表面积和

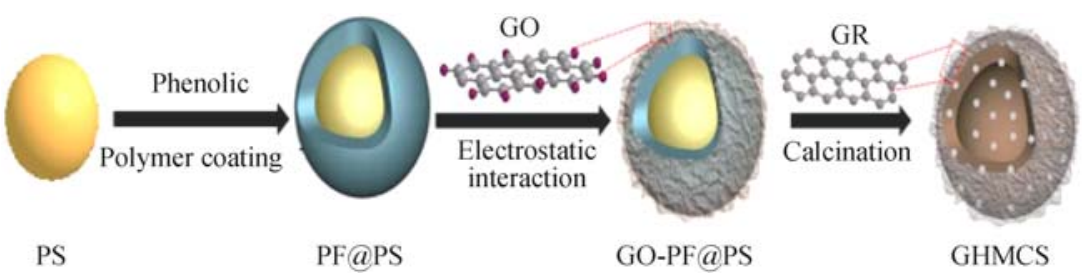

图 5 GHMCS 合成路线示意图 ${ }^{[39]}$

Fig. 5 Schematic illustration of the synthetic route for GHMCS ${ }^{[39]}$

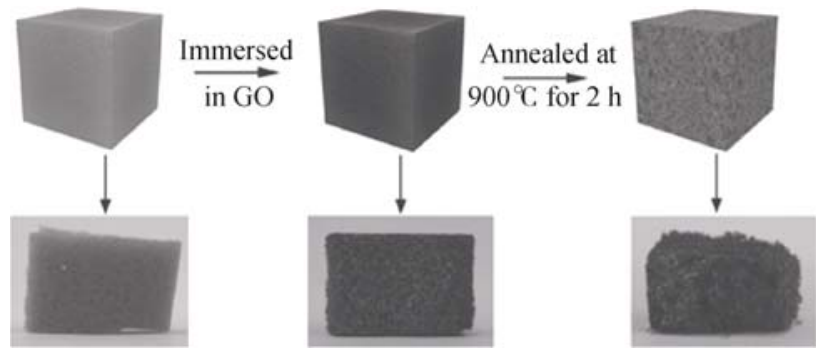

图 6 STGS 合成路线示意图 ${ }^{[40]}$

Fig. 6 Schematic representation of the procedure for preparing STGS samples ${ }^{[40]}$

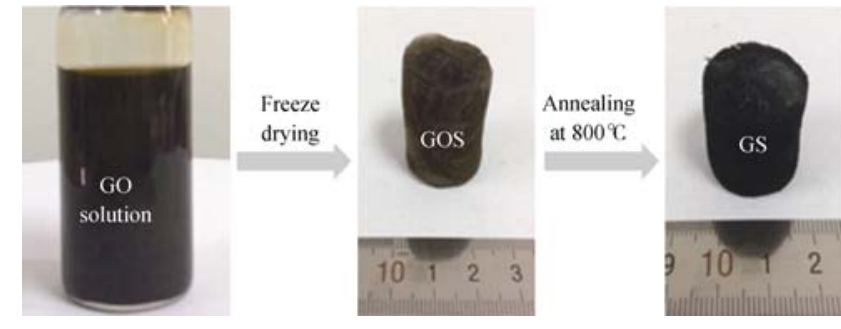

图 $7 \mathrm{GS}$ 合成路线示意图 ${ }^{[41]}$

Fig. 7 Schematic representation of the procedure for preparing GS sample $\mathrm{e}^{[41]}$
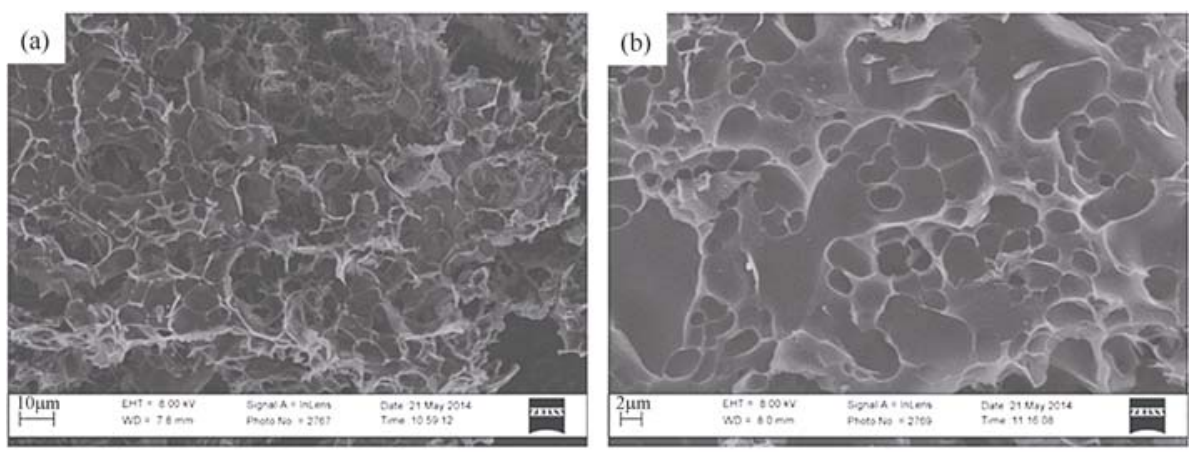

图 $8 \mathrm{KOH}$ 激发的石墨烯样品 SEM 照片 ${ }^{[46]}$

Fig. 8 SEM images $(a, b)$ of $\mathrm{KOH}$-activated graphene sample $\mathrm{e}^{[46]}$ 
导电率的目的 ${ }^{[42]}$ 。目前该方法已经被广泛地应用 于 $\mathrm{CNTS}^{[43]}$ 、碳纤维 ${ }^{[44]}$ 和石墨烯 ${ }^{[45]}$ 等碳材料。2015 年, $\mathrm{Li}$ 等 ${ }^{[46]}$ 首次将 $\mathrm{KOH}$ 激发的石墨烯应用于 $\mathrm{CDI}$ 技术，制备出含大量孔结构的三维石墨烯。从图 8 可以看出石墨烯为三维网络结构, 含大量孔结 构。测试表明, 该材料的比表面积达到 $3513 \mathrm{~m}^{2} / \mathrm{g}$, 导电率为 $104 \mathrm{~S} / \mathrm{m}$ 。基于此材料的 CDI 电极的电 吸附量达到 $11.86 \mathrm{mg} / \mathrm{g}$ 。以上数据充分表明利用 碱性物质激发石墨烯可以制备出性能优异的 CDI 电极材料。

从上面的论述可以看出, 与二维石墨烯相比, 三维石墨烯内部含有大量的贯通孔结构, 增大了材 料的比表面积和导电性能, 制备出的电极具有更好 的离子吸附性能, 更适合作为 CDI 电极材料。但目 前制备三维石墨烯的工艺较复杂、成本较高, 不利 于工业化推广, 此外, 制备的三维石墨烯孔径较大, 不利于吸附 $\mathrm{NaCl}$ 离子。

\section{2 石墨烯基复合物 CDI 电极的研究}

层一层之间的聚集效应，导致石墨烯的比表面 积远低于理论计算值。研究者们主要通过两种方法 解决该问题 ${ }^{[38]}$ : (1) 将石墨烯做成三维网络状, 增大 比表面积; (2)利用各种材料作为插入剂和分散剂, 如活性炭、碳纳米管、纳米金属颗粒和高分子聚合 物等, 增大石墨烯间距, 减少聚集效应, 达到增大 石墨烯比表面积的目的。下面将重点讨论石墨烯和 其他材料的复合对 CDI 电极性能的影响。

\section{1 石墨烯与多孔炭的复合}

活性炭(AC) 具有多孔结构, 其比表面积高达 $1000 \mathrm{~m}^{2} / \mathrm{g}$, 且价廉易得, 是 CDI 常用的电极材料, 但 $\mathrm{AC}$ 孔分布不均匀, 以微孔为主, 不利于吸附 $\mathrm{NaCl}$ 离子 ${ }^{[10-11,47]}$ 。 $\mathrm{Li}$ 等 ${ }^{[48]}$ 将氧化还原石墨烯 $(\mathrm{RGO})$ 与 $\mathrm{AC}$ 按不同比例复合, 制备出复合材料(GAC)。研究发现, 随着石墨烯掺量的增加, GAC 的比表面积持续下降, 同时微孔的体积会减少, 平均孔径会先增大后减小, 其中掺 20\%石墨烯的 GAC-20 的值最大, 为 $2.99 \mathrm{~nm}$, 此时石墨烯的比表面积达到 $779 \mathrm{~m}^{2} / \mathrm{g}$ 。CV 曲线如图 9 所示, 相同条件下, 基于 GAC-20 的电极的比电容 最大, 为 $181 \mathrm{~F} / \mathrm{g}$, 电吸附量为 $2.94 \mathrm{mg} / \mathrm{g}$, 高于 $\mathrm{AC}$ 和 $\mathrm{RGO}$ 的电极。 $\mathrm{GAC}$ 内, $\mathrm{RGO}$ 和 $\mathrm{AC}$ 相互交织在 一起, 构成网络结构, 一方面改变了 $\mathrm{AC}$ 孔的分布, 另一方面 $\mathrm{AC}$ 颗粒减少 RGO 的聚集作用, 加强 RGO 吸附离子的能力, 从而使电极表现出优异的性能。

与 $\mathrm{AC}$ 相比, 介孔碳( $\mathrm{MC}$ ) 拥有更大的孔径, 更 适合吸附 $\mathrm{NaCl}$ 离子, Zhang 等 ${ }^{[49]}$ 将热剥离制备的石
墨烯(GE)与 $\mathrm{MC}$ 复合, 制得复合材料 GE/MC。研究 发现, 掺入 $\mathrm{GE}$ 可增大材料的比表面积, $5 \% \mathrm{GE}$ 掺杂 量的 GE-5\%/MC 的比表面积为 $685.2 \mathrm{~m}^{2} / \mathrm{g}$, 高于 $\mathrm{MC}$ 的 $567.7 \mathrm{~m}^{2} / \mathrm{g}$, 且孔体积和平均孔径均有增加。CDI 电极性能测试表明, $\mathrm{GE} / \mathrm{MC}$ 电极比电容增加, 当扫 描速度为 $1 \mathrm{mV} / \mathrm{s}$ 时, $\mathrm{GE}-5 \% / \mathrm{MC}$ 电极为 $89.5 \mathrm{~F} / \mathrm{g}$, 而 $\mathrm{MC}$ 仅为 $72.55 \mathrm{~F} / \mathrm{g}$ 。此外, GE/MC 电极也表现出更 好的离子吸附性能, 如图 10 所示。

综上所述，将少量石墨烯与多孔碳复合，由于 协同作用，制备的复合材料同时具备石墨烯和多孔 碳的性能，具有良好导电性和较大比表面积。石墨 烯内含有孔径较大的片层状孔, 当石墨烯与多孔碳 复合时，可以调节多孔碳的孔径分布，提高电极的 电吸附能力; 而多孔碳作为插入剂和分散剂, 也可 在一定程度上缓解石墨烯的聚集效应，增强石墨烯 吸附离子的能力，使复合电极的性能好于纯的多孔 碳和石墨烯电极。但在石墨烯和多孔碳复合过程中, 当石墨烯含量较少时, 起作用的主要为多孔碳, 导

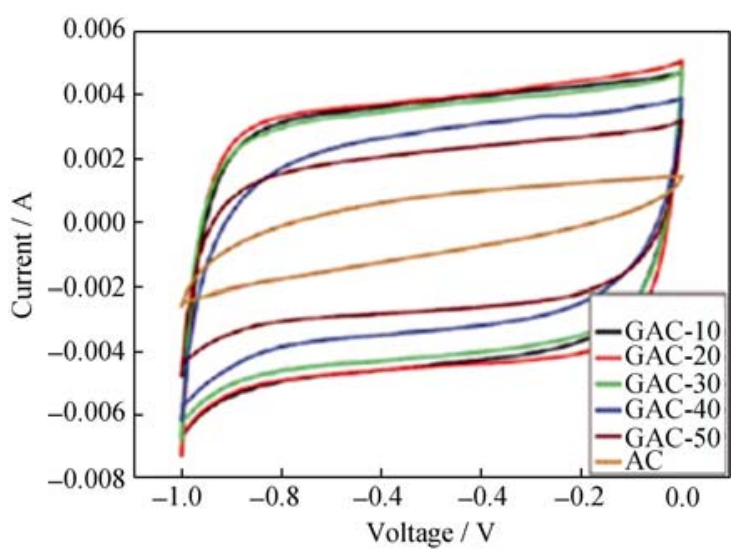

图 $9 \mathrm{NaCl}$ 中 $\mathrm{AC}$ 和 $\mathrm{GAC}$ 电极 $\mathrm{CV}$ 曲线(扫描速度 $1 \mathrm{mV} / \mathrm{s})^{[48]}$ Fig. $9 \mathrm{CV}$ curves of $\mathrm{AC}$ and $\mathrm{GAC}$ electrodes in $\mathrm{NaCl}$ solutions at a scan rate of $1 \mathrm{mV} / \mathrm{s}^{[48]}$

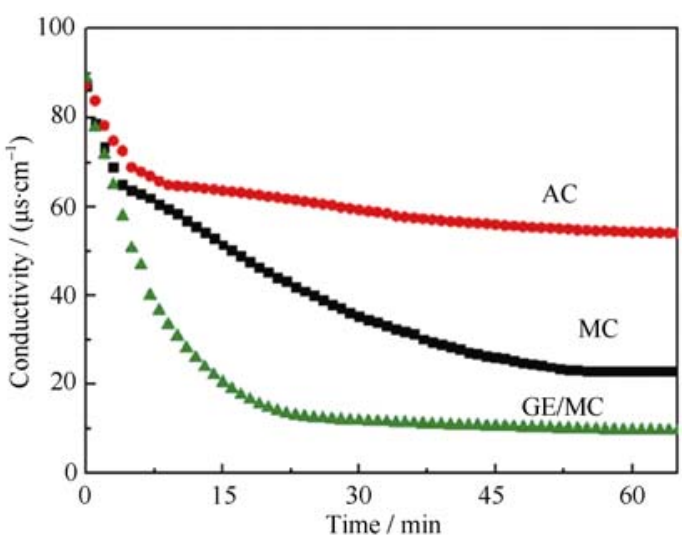

图 $10 \mathrm{AC} 、 \mathrm{MC}$ 和 $\mathrm{GE} / \mathrm{MC}$ 电极的 $\mathrm{CDI}$ 曲线对比图 ${ }^{[49]}$

Fig. 10 Comparison of CDI curves of the $\mathrm{AC}, \mathrm{MC}$ and GE/MC electrodes ${ }^{[49]}$ 
致电极的电吸附量较低; 而当石墨烯的含量较高时, 复合材料的比表面积往往会下降, 且电极的稳定性 也会受到影响 ${ }^{[48]}$ 。

\section{2 石墨烯与碳纳米管的复合}

碳纳米管 $(\mathrm{CNTs})$ 作为一种新型纳米材料, 具有 良好的导电性、较大的比表面积和良好的化学惰性, 一直被视作理想的水处理器件电极材料的研发对 象 ${ }^{[50-52]}$ 。石墨烯与碳纳米管复合时, CNTs 可以作为 分散剂和插入剂，使石墨烯分散更均匀。同时，石墨 烯的引入也可以增强碳纳米管电极的导电性。

如图 11 所示, CNTs 与 GR 复合时, CNTs 可进入 GR 层中, 使石墨烯进一步分散, 增大比表面积。 Zhang 等 ${ }^{[33]}$ 将不同量的 CNTs 加入到热剥离制备的 GR 中, 制备出网状复合材料 $(\mathrm{GR} / \mathrm{CNT})$ 。与 GR 相 比, 发现掺 CNTs 的 GR 的比表面积和气孔体积增 加。其中, $10 \% \mathrm{CNTs}$ 掺杂量的 GR/CNT- $10 \%$ 比表面 积为 $479.5 \mathrm{~m}^{2} / \mathrm{g}$, 提高了 $39.8 \%$, 气孔体积增加了 $32.9 \%$ 。同时，电极性能测试表明，GR/CNT-10\%电 极的比电容为 $68 \mathrm{~F} / \mathrm{g}$ (扫描速度为 $10 \mathrm{mV} / \mathrm{s}$ ), 高于 GR 电极的 $32 \mathrm{~F} / \mathrm{g}$ 。此外, GR/CNT-10\%电极的电吸 附量达到 $1.41 \mathrm{mg} / \mathrm{g}$, 而 GR 电极的值为 $1.10 \mathrm{mg} / \mathrm{g}$ 。 循环脱吸附实验则表明 GR/CNT 电极稳定性能更好, 如图 12 所示。 $\mathrm{Li}$ 等 ${ }^{[54]}$ 将少量的氧化还原石墨烯 (RGO)掺杂到 CNTs, 制备出 CNTs-RGO, 用来研究 不同石墨烯掺入量对 CNTs 电极性能的影响。他们 发现, 10\%掺入量的 CNTs-RGO-10 制备的电极材料的 性能最好, 其比电容为 $311.1 \mathrm{~F} / \mathrm{g}$ (扫描速度 $10 \mathrm{mV} / \mathrm{s}$ ), 大于 $\mathrm{CNTs}$ 电极的 $202 \mathrm{~F} / \mathrm{g}$ 和 $\mathrm{RGO}$ 电极的 $293.4 \mathrm{~F} / \mathrm{g}$ 。 以上实验均说明了石墨烯和碳纳米管复合, 可以制 备出性能优异的复合电极材料。

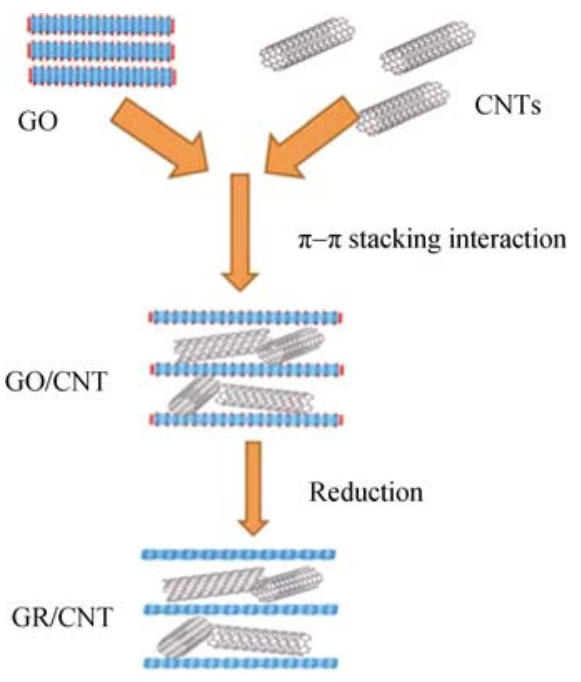

图 11 GR/CNT 合成示意图 ${ }^{[53]}$

Fig. 11 Preparation process of GR/CNT composites ${ }^{[53]}$

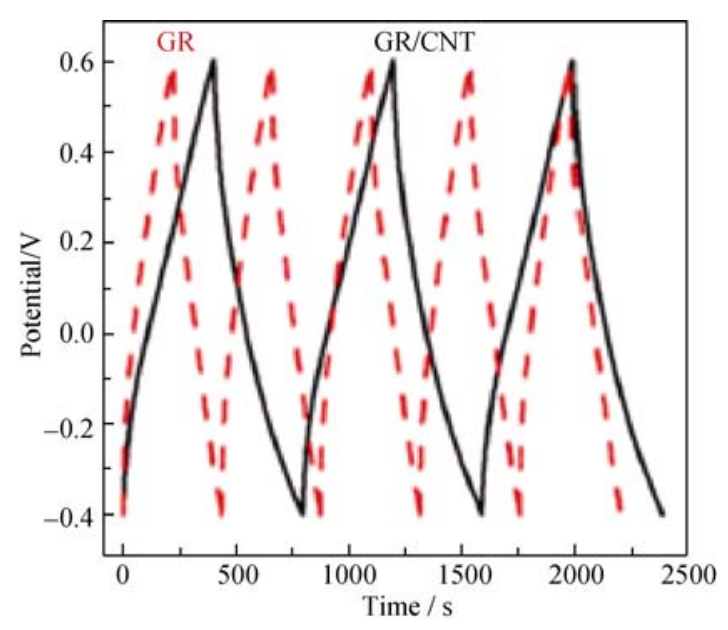

图 $12 \mathrm{GR} / \mathrm{CNT}$ 和 $\mathrm{GR}$ 电极充放电曲线 ${ }^{[53]}$

Fig. 12 Charge/discharge cycling curves of the GR and GR/CNT electrodes ${ }^{[53]}$

Wimalasiri 和 Zou ${ }^{[55-56]}$ 等按 10:1 的比例将氧化 石墨烯 GO 和单壁碳纳米管(SWCNTs)混合，利用水 合肼进行还原处理，制备出复合材料 $\mathrm{CNT} / \mathrm{G}$, 并将 其应用于 CDI 电极。值得注意的是, CNT/G 材料的 比表面积为 $391 \mathrm{~m}^{2} / \mathrm{g}$ ，与之前报道的数据类似，但 电极的电吸附量达到了 $26.42 \mathrm{mg} / \mathrm{g}$, 比电容达到 $213 \mathrm{~F} / \mathrm{g}$ (扫描速度 $10 \mathrm{mV} / \mathrm{s}$ ), 远大于之前报道的值。

Wimalasiri 和 Zou 将其归功于 $\mathrm{CNT} / \mathrm{G}$ 复合材料制备 工艺的不同。

碳纳米管可以提高石墨烯的导电性及比表面积, 二者结合可形成网状复合电极材料。基于该复合材 料的 CDI 电极的电吸附量较大, 表现出优异的电化 学性能。因此, 将石墨烯与碳纳米管进行复合是一 种提高电极性能的可行途径。

\section{3 石墨烯与纳米金属颗粒的复合}

为了改善石墨烯的分散性，可将高电容的纳米 金属颗粒作为分散物添加到石墨烯层中，从而达到 增大比表面积的目的。大量研究表明以石墨烯和纳 米金属颗粒的复合物作为电极材料, 可以提高超级 电容器比电容 ${ }^{[57-58]}$, 也从侧面论证了石墨烯和纳米 金属复合物增强 CDI 电极性能的可行性。

二氧化锡 $\left(\mathrm{SnO}_{2}\right)$ 是一种价格低廉, 环境友好型 的氧化物金属, 且具有良好的化学稳定性和高电 容, 已经在锂离子电池电极和传感器领域表现出 了优异的应用前景 ${ }^{[59]}$ 。El-Deen 等 ${ }^{[60]}$ 将纳米 $\mathrm{SnO}_{2}$ 与石墨烯复合, 采用 “一锅合成法” 制备出新型复 合 $\mathrm{CDI}$ 电极材料 $\mathrm{Gr} / \mathrm{SnO}_{2}$ 。经测试, 当 $\mathrm{SnO}_{2}$ 掺量 为 $15 \%$ 时, 电极表现出最好的性能, 其比电容为 $323 \mathrm{~F} / \mathrm{g}$ (扫描速度 $5 \mathrm{mV} / \mathrm{s})$, 脱盐率达到 $83 \%$, 电吸 
附量为 $1.49 \mathrm{mg} / \mathrm{g}$ 。证明了纳米金属颗粒可以减弱 石墨烯的聚合作用，增大石墨烯比表面积。石墨烯 与金属颗粒的复合，为提高石墨烯基 CDI 电极性 能提供了一种新的方法。

之后, El-Deen 等 ${ }^{[61]}$ 又研究了不同形状的纳米 $\mathrm{MnO}_{2}$ 对石墨烯 CDI 电极的影响。他们将颗粒状纳 米 $\mathrm{MnO}_{2}\left(\mathrm{MnO}_{2}-\mathrm{NPs}\right)$ 和棒状纳米 $\mathrm{MnO}_{2}\left(\mathrm{MnO}_{2}-\mathrm{NRs}\right)$ 分别与石墨烯复合, 制备出复合电极材料 $\mathrm{MnO}_{2}$ $\mathrm{NPs} @ \mathrm{GR}$ 和 $\mathrm{MnO}_{2}-\mathrm{NRs} @ \mathrm{GR}$ 。对比发现 $\mathrm{MnO}_{2}-\mathrm{NRs}$ 加强石墨烯分散程度的效果更明显, CDI 电极表现 出更好的性能。 $\mathrm{MnO}_{2}$-NRs@GR 电极的比电容达到 $292 \mathrm{~F} / \mathrm{g}$, 电吸附量为 $5.01 \mathrm{mg} / \mathrm{g}$, 脱盐率为 $93 \%$ 。从 图 13 可以看出 $\mathrm{GR} 、 \mathrm{AC}$ 以及 $\mathrm{MnO}_{2}-\mathrm{NPs} @ \mathrm{GR}$ 电极 的离子吸附能力和 $\mathrm{MnO}_{2}-\mathrm{NRs} @ \mathrm{GR}$ 电极的对比。

金属银 $(\mathrm{Ag})$ 具有良好的热稳定性、导电性 ${ }^{[62]}$ 和杀 菌功能 ${ }^{[63]}$, 为了提高 $\mathrm{CDI}$ 电极的电吸附性能和杀菌性 能, Cai 等 ${ }^{[64]}$ 将纳米 $\mathrm{Ag}$ 添加到石墨烯中, 制备出复合 $\mathrm{CDI}$ 电极材料 $\mathrm{Ag} / \mathrm{rGO}$ 。此外, 他们还通过 $\mathrm{Ag}^{+}-\beta$-环糊

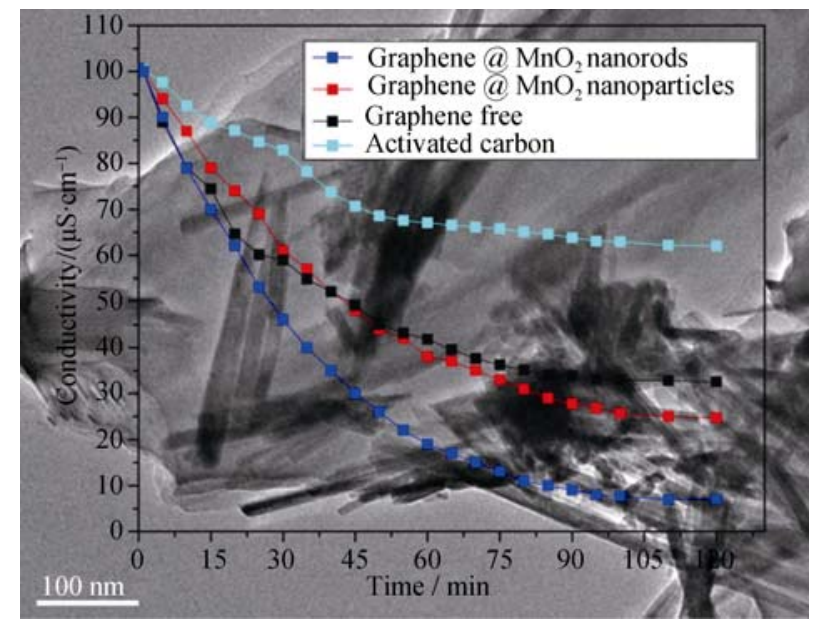

图 13 不同电极的 CDI 曲线对比图 ${ }^{[61]}$

Fig. 13 Comparison of CDI curves of different electrodes ${ }^{[61]}$
精 $(\mathrm{CD})$ 的炭化过程, 制备出 $\mathrm{Ag} @ \mathrm{C}$ 纳米颗粒, 并将其 与石墨烯复合制备出 $\mathrm{Ag} @ \mathrm{C} / \mathrm{rGO}$ 电极材料。电极性能 测试表明, $\mathrm{Ag} / \mathrm{rGO}$ 和 $\mathrm{Ag} @ \mathrm{C} / \mathrm{rGO}$ 电极可以减少充电 阻力, 电吸附性能更好。脱吸附时, $5 \mathrm{~min}$ 内电极可以 恢复到 $90 \%$ 以上，且 $\mathrm{Ag} / \mathrm{rGO}$ 和 $\mathrm{Ag} @ \mathrm{C} / \mathrm{rGO}$ 电极可以 明显地增加抗菌能力, 减少细菌的滋生。

二氧化钛 $\left(\mathrm{TiO}_{2}\right)$ 是一种优质的金属氧化物，具 有低毒、高稳定性和高介电常数等性质, 是一种理 想的增强石墨烯电极性能的金属材料 ${ }^{[65]}$ 。Yin 等 ${ }^{[66]}$ 以 $\mathrm{TiCl}_{3}$ 和 $\mathrm{GO}$ 为前驱物, $\mathrm{PVP}$ 为稳定剂, 将三维石 墨烯和 $\mathrm{TiO}_{2}$ 纳米颗粒复合, 制备出复合电极材料 $\mathrm{GA} / \mathrm{TiO}_{2}$, 其合成路线如图 14 所示。经测试, 当 $\mathrm{NaCl}$ 初始浓度为 $500 \mathrm{mg} / \mathrm{L}$ 时, $\mathrm{GA} / \mathrm{TiO}_{2}$ 电极的电吸 附量为 $15.1 \mathrm{mg} / \mathrm{g}$, 分别是 $\mathrm{GA}$ 电极的 1.5 倍, $\mathrm{AC}$ 电 极的 12.6 倍。 2015 年, El-Deen 等 ${ }^{[67]}$ 将 $\mathrm{TiO}_{2}$ 与石墨 烯复合制备出复合材料 $\left(\mathrm{rGO} / \mathrm{TiO}_{2}\right)$, 并将其应用于 膜电容去离子技术(MCDI)中，发现掺 $20 \% \mathrm{TiO}_{2}$ 的复 合电极的比电容达到 $443 \mathrm{~F} / \mathrm{g}$ (扫描速度 $10 \mathrm{mV} / \mathrm{s}$ ), 为 $\mathrm{rGO}$ 电极的 9 倍。工作电压为 $0.8 \mathrm{~V}$, 初始液浓度 为 $300 \mathrm{mg} / \mathrm{L}$, 复合电极的电吸附量达到 $9.1 \mathrm{mg} / \mathrm{g}$, 表现出优异的 CDI 电极性能。

综上所述，纳米金属作为分散物质，可以很好 地使石墨烯均匀分散, 达到增强 CDI 电极性能的目 的。不同的纳米金属, 不同的形状均会影响复合电 极的性能, 其中棒状纳米颗粒作用效果更好。将纳 米金属与三维石墨烯复合, 有望得到性能更加优异 的石墨烯基复合电极材料。但是以上研究未考虑纳 米金属颗粒在盐溶液中的稳定性对电极性能的影响; 脱盐过程中，纳米金属颗粒可能部分溶解于溶液中， 污染溶液。此外，纳米金属颗粒的存在也可能促进 水的电解反应和石墨烯表面的氧化反应等, 进一步 影响复合电极的电化学性能和稳定性。

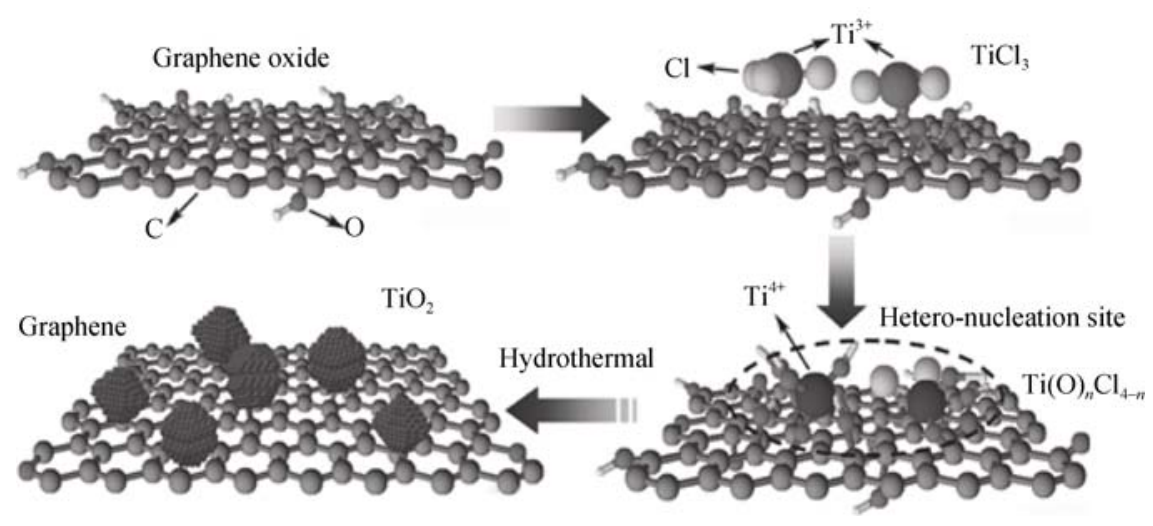

图 $14 \mathrm{GA} / \mathrm{TiO}_{2}$ 合成路线示意图 ${ }^{[66]}$

Fig. 14 Schematic illustration of the synthetic route for $\mathrm{GA} / \mathrm{TiO}_{2}{ }^{[66]}$ 


\section{4 石墨烯与聚合物的复合}

一些特殊的导电高分子聚合物, 如聚苯胺 (PANI), 因其具有高导电性和易合成性 ${ }^{[68-70]}$, 成为 研究热点。将导电聚合物与多孔碳材料复合是制备 复合电极材料的重要方法, 制备的复合电极的电化 学性能更佳, 但目前该复合电极主要是应用于锂离 子电池和超级电容器等。

Yan 等 ${ }^{[71}$ 将石墨烯与 PANI复合, 通过原位聚合 法制备出复合电极材料 G/PANI。他们将石墨烯与 PANI 单体苯胺混合, 苯胺在过硫酸铵作用下, 经聚 合作用合成 PANI, 合成的 PANI 与石墨烯相结合, 形成 G/PANI 复合材料, 合成路线如图 15 所示。 $\mathrm{N}_{2}$ 吸附测试表明 G/PANI 比表面积为 $394 \mathrm{~m}^{2} / \mathrm{g}$, 低于石 墨烯的 $428 \mathrm{~m}^{2} / \mathrm{g}$, 但 G/PANI 的孔径分布在 2 6 nm, 更适合吸附离子 ${ }^{[52]}$ 。从图 16 可看出 $\mathrm{G} / \mathrm{PANI}$ 电极的 电化学性能更佳, 比电容更大, 图 17 则表明了 G/PANI 电极的循环再生性能。由此说明 G/PANI 电 极具有更好的电极性能, 石墨烯与聚合物 PANI 复 合可以制备出性能优异的电极材料。
目前，石墨烯与聚合物复合用于 CDI 电极的报 道较少。但从其在超级电容中的应用 ${ }^{[69,72]}$ 和 Yan 等 ${ }^{[71]}$ 的研究可以看出, 导电性聚合物可以增强复合电极 的导电性, 加快离子输运速率, 提高电极比电容和 离子脱吸附速率。导电聚合物作为分散剂和插入剂, 可以增大石墨烯层间距，增大其比表面积，但是导 电高分子也可能会使石墨烯内部分气孔堵塞，使储 存离子的空位减少。此外, 当导电高聚物浓度过高 时，其分散不均匀，可能会形成团聚体，影响电极 的导电性。

\section{5 石墨烯与碳纤维的复合}

利用静电纺丝技术，可以制备出比表面积大、 导电性能良好的多孔纳米碳纤维网，将其用于 CDI 电极材料, 已经表现出优异的电极性能 ${ }^{[73-74]}$ 。石墨 烯具有较多的中孔和优异的导电性能，与碳纤维复 合, 可以调节材料孔径分布, 制备出性能更加优异 的 CDI 电极材料。

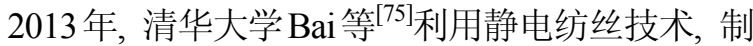
备出掺少量石墨的多孔碳纤维 GO-PCNF 和无石墨烯

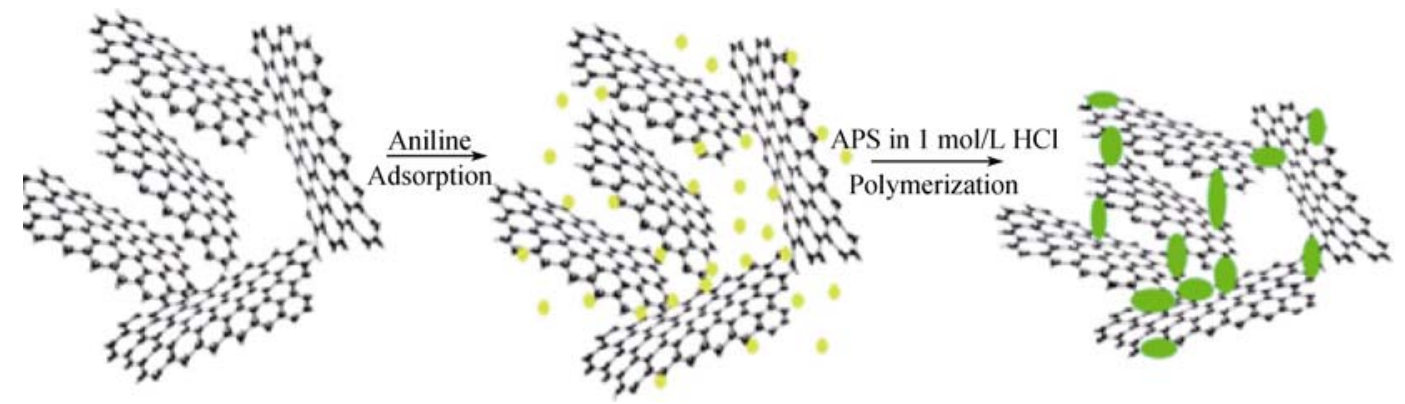

图 15 原位聚合法制备 PANI 和石墨烯复合物 ${ }^{[71]}$

Fig. 15 The in-situ polymerization of PANI in the presence of graphene sheets ${ }^{[71]}$

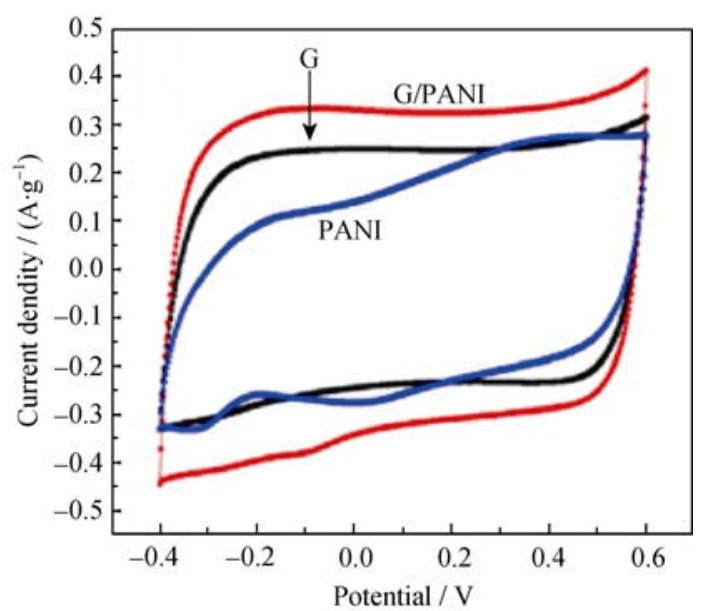

图 16 G、PANI 和 G/PANI 电极的 CV 曲线 ${ }^{[71]}$

Fig. $16 \mathrm{CV}$ curves of grapheme only, PANI only and G/ $\mathrm{PANI}^{[71]}$

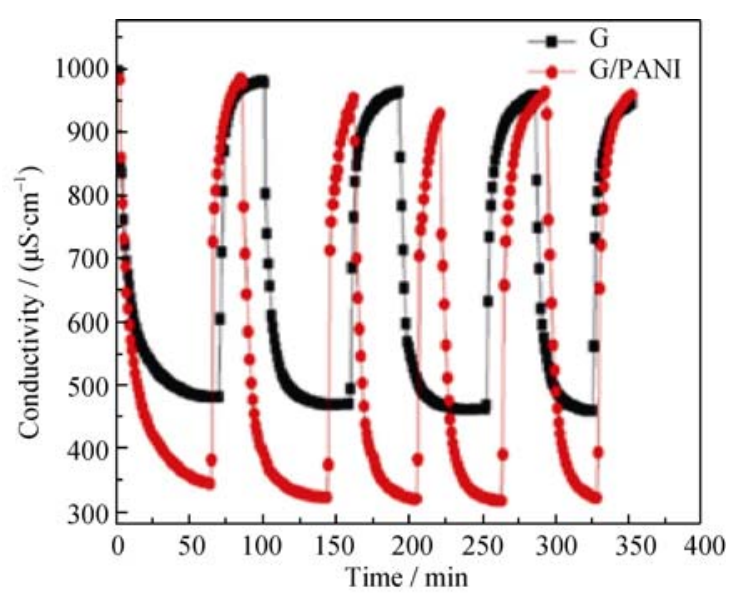

图 17 电极的脱吸附曲线 ${ }^{[71]}$

Fig. 17 Regeneration of electrodes performance ${ }^{[71]}$ 
的 PCNF 网状材料。研究发现, 石墨烯的引入使材料的 比表面积减小了 $18.7 \%$, 但中孔的比率增加了 $18 \%$, 更 有利于 $\mathrm{NaCl}$ 离子的吸附。电极性能测试发现, GO-PCNF 和 PCNF 电极均具有较高的离子吸附能力。 当初始浓度为 $100 \mathrm{mg} / \mathrm{L}$ 时, GO-PCNF 电极的电吸附 量为 $7.8 \mathrm{mg} / \mathrm{g}$, PCNF 电极为 $5.9 \mathrm{mg} / \mathrm{g}$, 所以 GO-PCNF 电极的离子吸附性能会更好一些。电极的比电容测试 也表明 GO-PCNF 电极的值较高, 为 $156.1 \mathrm{~F} / \mathrm{g}$ 。

Dong 等 ${ }^{[76]}$ 利用静电纺丝技术将不同量的氧化 还原石墨烯(RGO)与活性碳纤维 (ACF)复合, 制备 出 RGO/ACF 网状复合物。研究发现, RGO 的掺入 可以改善孔径分布, 增强电极性能。其中掺 $10 \%$ RGO 的 RGO/ACF-10 电极显示出了最佳的离子吸附能力, 电吸附量为 $7.2 \mathrm{mg} / \mathrm{g}$, 比 ACF 电极高 $53 \%$ 。

综上所述, 利用静电纺丝技术, 将石墨烯与纳
米碳纤维复合, 可以制备出性能优异的 CDI 电极材 料。这是由于石墨烯的引入, 改善了材料的孔径分 布，增大了中孔的比率。但是目前的研究中石墨烯 的掺入量较低, 未能充分发挥石墨烯优异的性能, CDI 电极性能还有待提高。

\section{3 总结}

由于石墨烯的聚集效应，导致比表面积仅为 $\sim 80 \mathrm{~m}^{2} / \mathrm{g}$, 远低于理论值, 故二维石墨烯薄层 CDI 电极效果并不好, 三维石墨烯具有独特的贯通孔结 构, 可以增大比表面积, 改善电极性能。石墨烯与 $\mathrm{AC} 、 \mathrm{MC} 、 \mathrm{CNFs}$ 和碳纳米纤维等复合可显著改善孔 径的分布, 提高电极的电吸附性能。目前, 石墨烯及 其复合物在 CDI 海水淡化领域中已经取得了一定的

表 1 不同石墨烯基电极材料的性能对比

Table 1 Comparison of the performance among different graphene-based electrode materials

\begin{tabular}{|c|c|c|c|c|c|c|}
\hline $\begin{array}{l}\text { Electrode } \\
\text { materials }\end{array}$ & $\begin{array}{l}\text { Specific surface } \\
\text { area } /\left(\mathrm{m}^{2} \cdot \mathrm{g}^{-1}\right)\end{array}$ & $\begin{array}{c}\text { [Specific capacitance/ } \\
\left.\left(\mathrm{F} \cdot \mathrm{g}^{-1}\right)\right] /[\text { Scan rate/ } \\
\left.\left(\mathrm{mV} \cdot \mathrm{s}^{-1}\right)\right]\end{array}$ & $\begin{array}{c}\text { Applied } \\
\text { voltage/V }\end{array}$ & $\begin{array}{c}\text { [Initial concentration/ } \\
\left.\left(\mathrm{mg} \cdot \mathrm{L}^{-1}\right)\right] /[\text { Initial con- } \\
\left.\text { ductivity } /\left(\mu \mathrm{S} \cdot \mathrm{cm}^{-1}\right)\right]\end{array}$ & $\begin{array}{c}\text { Electrosorption } \\
\text { capacity } /\left(\mathrm{mg} \cdot \mathrm{g}^{-1}\right)\end{array}$ & Ref. \\
\hline GR & 14.2 & $75.18 / 70.00$ & 2.0 & $-/ \sim 50$ & 1.85 & {$[21]$} \\
\hline GR & 77.0 & - & 2.0 & $22.8 /-$ & 0.46 & {$[17]$} \\
\hline GR & 222.1 & - & 2.0 & $-/ \sim 55$ & 1.35 & {$[26]$} \\
\hline GR & - & - & 2.0 & $-/ 86.9$ & 0.88 & {$[27]$} \\
\hline GR & 464.0 & $149.8 / 5.0$ & 2.0 & $-/ 500$ & 8.60 & [19] \\
\hline RGO-RF & 406.4 & $135.7 / 10.0$ & 2.0 & $-/ \sim 58$ & 1.42 & [35] \\
\hline 3DMGA & 339.0 & $58.4 / 5.0$ & 2.0 & $-/ \sim 105$ & 5.39 & [37] \\
\hline 3DGHPC & 384.4 & $80.34 / 10.00$ & 1.2 & $-/ 60$ & 6.18 & [38] \\
\hline GHMCS & 400.4 & $43.22 / 10.00$ & 1.6 & $-/ 68.5$ & 2.30 & [39] \\
\hline STGS & 305.0 & $57 / 10$ & 1.5 & $-/ \sim 106$ & 4.95 & [40] \\
\hline GS & 356.0 & $205.2 / 5.0$ & 1.2 & $500 /-$ & 14.90 & [41] \\
\hline $\begin{array}{c}\text { KOH-activated } \\
\text { GR }\end{array}$ & 3513.0 & - & 2.0 & $70 / 150$ & 11.86 & [46] \\
\hline $20 \% \mathrm{GR}+\mathrm{AC}$ & 779.0 & $181 / 1$ & 1.2 & $-/ 100$ & 2.94 & [48] \\
\hline $5 \% \mathrm{GR}+\mathrm{MC}$ & 685.2 & $89.5 / 1.0$ & - & $-/ \sim 90$ & 0.73 & [49] \\
\hline $\mathrm{GR}+10 \% \mathrm{CNTs}$ & 479.5 & $68 / 10$ & 2.0 & $-/ 57$ & 1.41 & {$[53]$} \\
\hline $10 \% \mathrm{GR}+\mathrm{CNTs}$ & 438.6 & $311.1 / 10.0$ & 1.6 & $-/ 100$ & 0.88 & {$[54]$} \\
\hline $\mathrm{GR}+10 \% \mathrm{SWCNTs}$ & 391.0 & $213 / 10$ & 2.0 & $780 / 1540$ & 26.42 & {$[55]$} \\
\hline $\mathrm{GR}+15 \% \mathrm{SnO}_{2}$ & - & $323 / 5$ & 1.4 & $-/ \sim 61$ & 1.49 & {$[60]$} \\
\hline $\mathrm{GR}+\mathrm{MnO}_{2}-\mathrm{NPs}$ & - & $180 / 10$ & 1.2 & $-/ \sim 100$ & $\sim 3.50$ & {$[61]$} \\
\hline $\mathrm{GR}+\mathrm{MnO}_{2}-\mathrm{NRs}$ & - & $292 / 10$ & 1.2 & $-/ \sim 100$ & 5.01 & {$[61]$} \\
\hline $\mathrm{GR}+\mathrm{Ag}$ & - & $114.7 / 25.0$ & 1.5 & $-/-$ & - & [64] \\
\hline GR+Ag@C & - & $107.6 / 25.0$ & 1.5 & $-/-$ & - & [64] \\
\hline $\mathrm{GR}+\mathrm{TiO}_{2}$ & 187.6 & $142.6 / 5.0$ & 1.2 & $500 /-$ & 15.10 & {$[66]$} \\
\hline $\mathrm{GR}+20 \% \mathrm{TiO}_{2}$ & - & $443 / 10$ & 0.8 & $\sim 300 /-$ & 9.10 & [67] \\
\hline $\mathrm{GR}+4 \% \mathrm{PANI}$ & 394.0 & - & 1.2 & $500 /-$ & - & {$[71]$} \\
\hline $\mathrm{GR}+\mathrm{PCNF}$ & 474.0 & $151 /-$ & 1.2 & $100 /-$ & 7.80 & {$[75]$} \\
\hline $10 \% \mathrm{GR}+\mathrm{ACF}$ & 621.0 & $193 / 5$ & 1.2 & $400 /-$ & 7.20 & {$[76]$} \\
\hline
\end{tabular}


进展，各种石墨烯基 CDI 电极性能对比如表 1 所示。 但目前的研究仍处于初始阶段, 仍存在一些需继续 深入研究的问题。

1) 制备的石墨烯材料质量不稳定。文中所涉及 的大多为氧化还原石墨烯, 其制备过程中不确定性 因素很多。相同条件制备的石墨烯比表面积、孔径 分布等差别较大, 导致制备的电极性能不稳定, 不 利于工业化推广。

2) CDI 电极的稳定性函待解决。需综合考虑影 响 CDI 电极的因素, 部分研究仅仅测试了电极的几 个循环脱吸附曲线, 便得出电极十分稳定的结论, 然而并无法保证 CDI 电极的长期稳定性。

3) 未考虑实际海水环境对 CDI 电极的影响。 目前仅利用 $\mathrm{NaCl}$ 溶液进行相关研究, 未考虑复杂 海水组分对 CDI 电极影响。

虽然目前存在许多技术挑战，但 CDI 技术作为 一种高效节能、绿色环保的脱盐工艺，仍然显示出 巨大的应用前景，不仅可以应用于海水淡化，还可 应用于重金属萃取、污水脱盐处理等领域。此外, CDI 技术可以通过选取合适复合电极材料, 实现选 择性除去目标离子的目的，应用于水软化等领域。

\section{参考文献:}

[1] SHANNON M A, BOHN P W, ELIMELECH M, et al. Science and technology for water purification in the coming decades. Nature, 2008, 452(7185): 301-310.

[2] ANDERSON M A, CUDERO A L, PALMA J. Capacitive deionization as an electrochemical means of saving energy and delivering clean water. Comparison to present desalination practices: will it compete? Electrochimica Acta, 2010, 55(12): 3845-3856.

[3] ZOU L, VIDALIS I, STEELE D, et al. Surface hydrophilic modification of RO membranes by plasma polymerization for low organic fouling. Journal of Membrane Science, 2011, 369(1/2): $420-428$.

[4] YANG C M, CHOI W H, NA B K, et al. Capacitive deionization of $\mathrm{NaCl}$ solution with carbon aerogel-silicagel composite electrodes. Desalination, 2005, 174(2): 125-133.

[5] Welgemoed T J, SCHUTTE C F. Capacitive deionization dechnology ${ }^{\mathrm{TM}}$ : an alternative desalination solution. Desalination, 2005, 183(1/2/3): 327-340.

[6] MURPHY G W, CAUDLE D D. Mathematical theory of electrochemical demineralization in flowing systems. Electrochimical Acta, 1967, 12: 1655-1664.

[7] JOHNSON A M, NEWMAN J. Desalting by means of porous carbon electrodes. Journal of the Electrochemical Society, 1971, 118(3): 510-517.
[8] FARMER J C, FIX D V, MACK G V, et al. Capacitive deionization of $\mathrm{NH}_{4} \mathrm{ClO}_{4}$ solutions with carbon aerogel electrodes. Journal of Applied Electrochemistry, 1996, 26(10): 1007-1018.

[9] FARMER J C, FIX D V, MACK G V, et al. Capacitive deionization of $\mathrm{NaCl}$ and $\mathrm{NaNO}_{3}$ solutions with carbon aerogel electrodes. Journal of the Electrochemical Society, 1996, 143(1): 159-169.

[10] XU P, DREWES J E, HEIL D, et al. Treatment of brackish produced water using carbon aerogel-based capacitive deionization technology. Water Research, 2008, 42(10/11): 2605-2617.

[11] VILLAR I, ROLDAN S, RUIZ V, et al. Capacitive deionization of $\mathrm{NaCl}$ solutions with modified activated carbon electrodes. Energy \& Fuels, 2010, 24(6): 3329-3333.

[12] ZOU L, MORRIS G, QI D. Using activated carbon electrode in electrosorptive deionisation of brackish water. Desalination, 2008, 225(1/2/3): 329-340.

[13] RASINES G, LAVELA P, MAC AS C, et al. Electrochemical response of carbon aerogel electrodes in saline water. Journal of Electroanalytical Chemistry, 2012, 671: 92-98.

[14] PENG Z, ZHANG D S, SHI L Y, et al. Comparative electroadsorption study of mesoporous carbon electrodes with various pore structures. The Journal of Physical Chemistry C, 2011, 115(34): 17068-17076.

[15] TSOURIS C, MAYES R, KIGGANS J, et al. Mesoporous carbon for capacitive deionization of saline water. Environment Science \& Technology, 2011, 45(23): 10243-10249.

[16] WANG L, WANG M, HUANG Z H, et al. Capacitive deionization of $\mathrm{NaCl}$ solutions using carbon nanotube sponge electrodes. Journal of Materials Chemistry, 2011, 21(45): 18295-18299.

[17] LI H B, PAN L K, LU T, et al. A comparative study on electrosorptive behavior of carbon nanotubes and graphene for capacitive deionization. Journal of Electroanalytical Chemistry, 2011, 653(1/2): $40-44$.

[18] ZHANG D S, SHI L Y, FANG J H, et al. Influence of diameter of carbon nanotubes mounted in flow-through capacitors on removal of $\mathrm{NaCl}$ from salt water. Journal of Materials Science, 2006, 42(7): $2471-2475$

[19] JIA B P, ZOU L. Graphene nanosheets reduced by a multi-step process as high-performance electrode material for capacitive deionisation. Carbon, 2012, 50(6): 2315-2321.

[20] WANG Z, YUE L, LIU Z T, et al. Functional graphene nanocomposite as an electrode for the capacitive removal of $\mathrm{FeCl}_{3}$ from water. Journal of Materials Chemistry, 2012, 22(28): 14101-14107.

[21] LI H B, LU T, PAN L K, et al. Electrosorption behavior of graphene in $\mathrm{NaCl}$ solutions. Journal of Materials Chemistry, 2009, 19(37): 6773-6779.

[22] SINGH V, JOUNG D, ZHAI L, et al. Graphene based materials: Past, present and future. Progress in Materials Science, 2011, 
56(8): $1178-1271$.

[23] STOLLER M D, PARK S, ZHU Y W, et al. Graphene-based ultracapacitors. Nano Letters, 2008, 8(10): 3498-3502.

[24] HUMMERS W, OFFEMA R. Preparation of graphitic oxide. Journal of the American Chemical Society, 1958, 80: 1339.

[25] XU Y X, BAI H, LU G W, et al. Flexible graphene films via the filtration of water-soluble. Journal of the American Chemical Society, 2008, 130: 5856-5857.

[26] LI H B, ZOU L, PAN L K, et al. Novel graphene-like electrodes for capacitive deionization. Environment Science \& Technology, 2010, 44: 8692-8697.

[27] WANG H, ZHANG D S, YAN T T, et al. Graphene prepared via a novel pyridine-thermal strategy for capacitive deionization. Journal of Materials Chemistry, 2012, 22(45): 23745-23748.

[28] JIA B P, ZOU L. Functionalized graphene as electrode material for capacitive deionization. Science of Advanced Materials, 2013, 5(8): 1111-1116.

[29] JIA B P, ZOU L. Wettability and its influence on graphene nansoheets as electrode material for capacitive deionization. Chemical Physics Letters, 2012, 548: 23-28.

[30] SI Y, SAMULSKI E T. Synthesis of water soluble graphene. Nano Letters, 2008, 8(6): 1679-1682.

[31] DREYER D R, PARK S , BIELAWSKI C W, et al. The chemistry of graphene oxide. Chemical Society Reviews, 2010, 39: 228-240.

[32] LIU Y, NIE C Y, LIU X J, et al. Review on carbon-based composite materials for capacitive deionization. RSC Advances, 2015, 5: $15205-15225$.

[33] WORSLEY M A, KUCHEYEV S O, SATCHER J H, et al. Mechanically robust and electrically conductive carbon nanotube foams. Applied Physics Letters, 2009, 94: 073115.

[34] LI C, SHI G. Three-dimensional graphene architectures. Nanoscale, 2012, 4(18): 5549-5563.

[35] WANG Z, DOU B J, ZHENG L, et al. Effective desalination by capacitive deionization with functional graphene nanocomposite as novel electrode material. Desalination, 2012, 299: 96-102.

[36] WORSLEY M A, PAUZAUSKIE P J, OLSON T Y, et al. Synthesis of graphene aerogel with high electrical conductivity. Journal of the American Chemical Society, 2010, 132: 14067-14069.

[37] WANG H, ZHANG D S, YAN T T, et al. Three-dimensional macroporous graphene architectures as high performance electrodes for capacitive deionization. Journal of Materials Chemistry A, 2013, 1(38): 11778-11789.

[38] WEN X R, ZHANG D S, YAN T T, et al. Three-dimensional graphenebased hierarchically porous carbon composites prepared by a dual-template strategy for capacitive deionization. Journal of Materials Chemistry A, 2013, 1(39): 12334-12344.

[39] WANG H, SHI L Y, YAN T T, et al. Design of graphene-coated hollow mesoporous carbon spheres as high performance electrodes for capacitive deionization. Journal of Materials Chemistry A, 2014, 2(13): 4739-4750.

[40] YANG Z Y, JIN L J, LU G Q, et al. Sponge-templated preparation of high surface area graphene with ultrahigh capacitive deionization performance. Advanced Functional Materials, 2014, 24(25): 3917-3925.

[41] XU X T, PAN L K, LIU Y, et al. Facile synthesis of novel graphene sponge for high performance capacitive deionization. Scientific Reports, 2015, 5: 8458 .

[42] LILLO-RODENAS M A, CAZORLA-AMOROS D, LINARESSOLANO A. Understanding chemical reactions between carbons and $\mathrm{NaOH}$ and $\mathrm{KOH}$ : an insight into the chemical activation mechanism. Carbon, 2003, 41: 267-275.

[43] RAYMUNDO-PINERO E, AZA1S P, CACCIAGUERRA T, et al. $\mathrm{KOH}$ and $\mathrm{NaOH}$ activation mechanisms of multiwalled carbon nanotubes with different structural organisation. Carbon, 2005, 43: 786-795.

[44] BARRANCO V, LILLO-RODENAS M A, LINARES-SOLANO A, et al. Amorphous carbon nanofibers and their activated carbon nanofibers as supercapacitor electrodes. Journal of Physical Chemistry C, 2010, 114: 10302-10307.

[45] ZHU Y W, MURALI S, STOLLER M D, et al. Carbon-based supercapacitors produced by activation of graphene. Science, 2011, 332: $1537-1541$.

[46] LI Z, SONG B, WU Z K, et al. 3D porous graphene with ultrahigh surface area for microscale capacitive deionization. Nano Energy, 2015, 11: 711-718.

[47] LEE J B, PARK K K, YOON S W, et al. Desalination performance of a carbon-based composite electrode. Desalination, 2009, 237(1): 155-161.

[48] LI H B, PAN L K, NIE C Y, et al. Reduced graphene oxide and activated carbon composites for capacitive deionization. Journal of Materials Chemistry, 2012, 22(31): 15556-15561.

[49] ZHANG D S, WEN X R, SHI L Y, et al. Enhanced capacitive deionization of graphene/mesoporous carbon composites. Nanoscale, 2012, 4(17): 5440-5446.

[50] GAO Y, PAN L K, ZHANG Y P, et al. Electrosorption of $\mathrm{FeCl}_{3}$ solution with carbon nanotubes and nanofibres film electrodes grown on graphite substrates. Surface Review Letters, 2007, 14(6): 1033-1037.

[51] LI H B, PAN L K, ZHANG Y P, et al. Ferric ion adsorption and electrodesorption by carbon nanotubes and nanofibres films. Water Science and Technology, 2009, 59(8): 1657-1663.

[52] PAN L K, WANG X Z, GAO Y, et al. Electrosorption of anions with carbon nanotube and nanofibre composite film electrodes. Desalination, 2009, 244(1/2/3): 139-143. 
[53] ZHANG D S, YAN T T, SHI L Y, et al. Enhanced capacitive deionization performance of graphene/carbon nanotube composites. Journal of Materials Chemistry, 2012, 22(29): 14696-14704.

[54] LI H B, LIANG S, LI J, et al. The capacitive deionization behaviour of a carbon nanotube and reduced graphene oxide composite. Journal of Materials Chemistry A, 2013, 1(21): 6335-6341.

[55] WIMALASIRI Y, ZOU L. Carbon nanotube/graphene composite for enhanced capacitive deionization performance. Carbon, 2013, 59: $464-471$.

[56] WIMALASIRI Y, ZOU L. Response to "Comments on 'carbon nanotube/graphene composite for enhanced capacitive deionization performance' by Y. Wimalasiri and L. Zou". Carbon, 2015, 81: 847-849.

[57] SUN X, XIE M, WANG G K, et al. Atomic layer deposition of $\mathrm{TiO}_{2}$ on graphene for supercapacitors. Journal of The Electrochemical Society, 2012, 159(4): A364.

[58] QIAN Y, LU S B, GAO F L. Preparation of $\mathrm{MnO}_{2} /$ graphene composite as electrode material for supercapacitors. Journal of Materials Science, 2011, 46(10): 3517-3522.

[59] SEEMA H, KEMP K C, CHANDRA V, et al. Graphene- $\mathrm{SnO}_{2}$ composites for highly efficient photocatalytic degradation of methylene blue under sunlight. Nanotechnology, 2012, 23: 355705.

[60] EL-DEEN A G, BARAKAT N A M, KHALIL K A, et al. Graphene/ $\mathrm{SnO}_{2}$ nanocomposite as an effective electrode material for saline water desalination using capacitive deionization. Ceramics International, 2014, 40(9): 14627-14634.

[61] EL-DEEN A G, BARAKAT N A M, KIM H Y. Graphene wrapped $\mathrm{MnO}_{2}$-nanostructures as effective and stable electrode materials for capacitive deionization desalination technology. Desalination, 2014, 344: 289-298.

[62] PASRICHA R, GUPTA S, SRIVASTAVA A K. A facile and novel synthesis of Ag-graphene-based nanocomposites. Small, 2009, 5(20): 2253-2259.

[63] ZHENG L, ZHANG G N, ZHANG M, et al. Preparation and capacitance performance of Ag-graphene based nanocomposite. Journal of Power Sources, 2012, 201: 376-381.

[64] CAI P F, SU C J, CHANG W T, et al. Capacitive deionization of seawater effected by nano $\mathrm{Ag}$ and $\mathrm{Ag} @ \mathrm{C}$ on graphene. Marine Pollution Bulletin, 2014, 85(2): 733-737.

[65] ZHU C Z, GUO S J, WANG P, et al. One-pot, water-phase ap- proach to high-quality graphene $/ \mathrm{TiO}_{2}$ composite nanosheets. Chemical Communications, 2010, 46(38): 7148-7150.

[66] YIN H J, ZHAO S L, WAN J W, et al. Three-dimensional graphene/ metal oxide nanoparticle hybrids for high-performance capacitive deionization of saline water. Advanced Materials, 2013, 25(43): 6270-6276

[67] EL-DEEN A G, CHOI J H, KIM C S, et al. $\mathrm{TiO}_{2}$ nanorod-intercalated reduced graphene oxide as high performance electrode material for membrane capacitive deionization. Desalination, 2015, 361: 53-64.

[68] LAI L F, YANG H P, WANG L, et al. Preparation of supercapacitor electrodes through selection of graphene surface functionalities. ACS Nano, 2012, 6(7): 5941-5951.

[69] ZHOU Y, QIN Z Y, LI L, et al. Polyaniline/multiwalled carbon nanotube composites with core-shell structures as supercapacitors electrode materials. Electrochimica Acta, 2010, 55(12): 3904-3908.

[70] LI Q. Application of polyaniline modified graphite electrodes for capacitive deionization of aqueous $\mathrm{NaCl}$ solution. Asian Journal of Chemistry, 2010, 22(10): 8126-8130.

[71] YAN C J, KANATHTHAGE Y W, SHORT R, et al. Graphene/ polyaniline nanocomposite as electrode material for membrane capacitive deionization. Desalination, 2014, 344: 274-279.

[72] WU Q, XU Y X, YAO Z Y, et al. Supercapacitors based on flexible graphene/polyaniline nanofiber composite films. ACS Nano, 2010, 4(4): 1963-1970.

[73] WANG M, HUANG Z H, WANG L, et al. Electrospun ultrafine carbon fiber webs for electrochemical capacitive desalination. New Journal of Chemistry, 2010, 34(9): 1843-1845.

[74] ZHOU Z P, WU X F, FONG H. Electrospun carbon nanofibers surface-grafted with vapor-grown carbon nanotubes as hierarchical electrodes for supercapacitors. Applied Physics Letters, 2012, 100: 023114-023115.

[75] BAI Y, HUANG Z H, YU X L, et al. Graphene oxide-embedded porous carbon nanofiber webs by electrospinning for capacitive deionization. Colloids and Surfaces A: Physicochemial and Engineering Aspects, 2014, 444: 153-158.

[76] DONG Q, WANG G, QIAN B, et al. Electrospun composites made of reduced graphene oxide and activated carbon nanofibers for capacitive deionization. Electrochimica Acta, 2014, 137: 388-394. 